\title{
Bacterial tRNA Modification Enzymes: Potential Role in Biology and Virulence
}

\section{Daniel C Shippy and Amin A Fadl*}

Department of Animal Sciences, University of Wisconsin-Madison, Madison, WI 53706, USA

RNA molecules contain four standard nucleosides, adenosine (A), guanosine $(\mathrm{G})$, cytidine $(\mathrm{C})$, and uridine $(\mathrm{U})$. Post-transcriptional RNA modifications are present in many types of RNAs including ribosomal RNA (rRNA), messenger RNA (mRNA), transfer RNA (tRNA), and others. These RNA modifications are important for altering the chemical and physical properties of nucleotides resulting in increased efficiency of RNA functions. Of all the RNAs, tRNA exhibits the largest number and most diverse modifications with cells from all kingdoms of life allocating a large portion of their genome to encoding enzymes involved in the post-transcriptional modification of nucleosides in tRNA [1]. Post-transcriptional modification of the anticodon domain in tRNA is a major factor in controlling gene expression which enables bacteria to survive in many different environments [2]. Modifications of uridine at the wobble position of the anticodon (U34) are required for the recognition of codons that are rarely used. In the absence of modifications, a shift in the translational reading frame occurs resulting in the expression of alternate protein sequences [2].

Numerous enzymes have been identified in the modification pathways of bacterial tRNAs with glucose-inhibited division (GidA) protein and MnmE being two of the enzymes investigated. GidA, also known as MnmG, was first described in Escherichia coli as a cell division protein because deletion of gidA resulted in a filamentous morphology when grown in a rich medium supplemented with glucose [3]. Further studies have suggested a role for GidA in the cell division and morphology of Salmonella enterica serovar Typhimurium (STM) and Aeromonas hydrophila [4,5]. Most importantly, studies in E. coli suggest GidA is a tRNA modification methylase responsible for the proper biosynthesis of 5-methylaminomethyl-2-thiouridine $\left(\mathrm{mnm}^{5} \mathrm{~s}^{2} \mathrm{u}\right)$ at the 5 position of the wobble uridine (U34) of tRNAs [6,7]. $\mathrm{MnmE}$, also known as TrmE, is a three domain protein proposed to be a molecular switch GTPase which assumes different conformations depending on whether it is bound to GTP or GDP [8]. In E. coli, MnmE is responsible for glutamate-dependent acid resistance by activating the transcriptional regulator gadE [9]. MnmE also appears to be a tRNA modification enzyme as MnmE deletion mutants are defective in the synthesis of $\mathrm{mnm}^{5} \mathrm{~s}^{2} \mathrm{u}[6]$.

In E. coli, it has been suggested that GidA and MnmE are part of the same tRNA modification pathway [6,7]. The study by Yim et al. [10] reported that mutations in E. coli gidA impaired the biosynthesis of $\mathrm{mnm}^{5} \mathrm{~s}^{2} \mathrm{u}$. Their study also showed identical levels of the same undermodified form of U34 are present in tRNA hydrolysates from gidA and mnmE mutants suggesting GidA and MnmE form a functional complex in which both proteins are interdependent. Further studies done in E. coli have provided additional evidence suggesting the in vitro binding ability of GidA and $\mathrm{MnmE}$ and that together these two enzymes are responsible for the proper biosynthesis of $\mathrm{mnm}^{5} \mathrm{~s}^{2} \mathrm{u}$ in bacterial tRNA [11-13]. Additionally, Shippy et al. [14] showed that GidA and MnmE bind together to modify Salmonella tRNA.

One of the most interesting aspects of the GidA/MnmE tRNA modification pathway is its potential role as a pathogenic regulatory mechanism. Studies have shown that deletion of gidA attenuates virulence and alters the biological characteristics of some bacteria
[5,14-18]. In Myxococcus xanthus, GidA is described as a flavoprotein involved in fruiting body development [15]. The study by Kinscherf and Willis [16] suggests GidA is a global regulator of Pseudomonas syringae, as deletion of gidA affected numerous phenotypic traits. GidA has also been found to regulate a potent virulence factor of $A$. hydrophila, the cytotoxic enterotoxin (ACT) [5]. Furthermore, GidA was found to regulate $r h l$ quorum sensing via RhlR-dependent and RhlR-independent pathways in Pseudomonas aeruginosa [17]. In Salmonella, deletion of gidA significantly attenuated both in vitro and in vivo virulence, and GidA was identified as a potential regulator of numerous genes and proteins associated with Salmonella pathogenicity island (SPI)-1 and SPI-2 [18]. Shippy et al. [14] have also implicated a role for MnmE in bacterial virulence. Their study reported deletion of $\mathrm{MnmE}$ attenuated the in vitro and in vivo virulence of Salmonella, but not to the extent seen in a gidA deletion mutant. A gidA mnmE double deletion mutant, however, was more attenuated than a single gidA or mnmE deletion mutant [14].

A major benefit of these attenuated bacterial strains is their potential use in live-attenuated vaccines. The study by Shippy and Fadl [19] characterized a gidA deletion mutant for potential use in a live-attenuated Salmonella vaccine. In their study, vaccination with a gidA deletion mutant fully protected mice from challenge with a highly lethal dose of the wild-type Salmonella strain. Both cellular and antibody mediated immunity were elicited as part of the protective mechanism provided by vaccination with a gidA deletion mutant [19]. Another study by Cho et al. shows tRNA modification by GidA is essential for Streptococcus pyogenes virulence, and suggests deletion of genes encoding tRNA modification enzymes as a new strategy to make avirulent strains for use in live-attenuated vaccines [20].

Overall, the GidA/MnmE tRNA modification pathway appears to be part of a major virulence mechanism in bacteria. Future work is needed to identify and characterize other enzymes potentially involved in this pathway as well as other pathways GidA and MnmE are associated with. Studies are needed in order to determine how GidA and $\mathrm{MnmE}$ regulate the specific genes and proteins identified for the pathogenic processes studied. Investigation into a gidA $m n m E$ double deletion mutant for use in a live-attenuated vaccine, or as a vaccine vector, could lead to a promising therapeutic strategy to control or prevent disease.

*Corresponding author: Amin A Fadl, Department of Animal Sciences, University of Wisconsin-Madison, 1675 Observatory Drive, Madison, WI 53706, USA, Tel(608) 890-2398 ; Fax: (608) 262-5157; E-mail: fadl@wisc.edu

Received December 06, 2013; Accepted December 08, 2013; Published January 10,2014

Citation: Shippy DC, Fadl AA (2014) Bacterial tRNA Modification Enzymes: Potential Role in Biology and Virulence. Biol Med 6: e106. doi: 10.4172/0974 8369.1000e105

Copyright: (c) 2014 Shippy DC. This is an open-access article distributed under the terms of the Creative Commons Attribution License, which permits unrestricted use, distribution, and reproduction in any medium, provided the original author and source are credited. 
Citation: Shippy DC, Fadl AA (2014) Bacterial tRNA Modification Enzymes: Potential Role in Biology and Virulence. Biol Med 6: e106. doi: 10.4172/0974-8369.1000e105

Page 2 of 2

\section{References}

1. Agris PF, Vendeix FA, Graham WD (2007) tRNA's wobble decoding of the genome: 40 years of modification. J Mol Biol 366: 1-13.

2. Gustilo EM, Vendeix FA, Agris PF (2008) tRNA's modifications bring order to gene expression. Curr Opin Microbiol 11: 134-40.

3. von Meyenburg K, Jorgensen BB, Nielsen J, Hansen F (1982) Promoters of the atp operon coding for the membrane-bound ATP synthase of Escherichia coli mapped by Tn10 insertion mutations. Mol Gen Genet 188: 240-8.

4. Shippy DC, Heintz JA, Albrecht RM, Eakley NM, Chopra AK, Fadl AA (2012) Deletion of glucose-inhibited division (gidA) gene alters the morphological and replication characteristics of Salmonella enterica Serovar typhimurium. Arch Microbiol 194: 405-12.

5. Sha J, Kozlova EV, Fadl AA, Olano JP, Houston CW, Peterson JW, et al (2004) Molecular characterization of a glucose-inhibited division gene, gidA, that regulates cytotoxic enterotoxin of Aeromonas hydrophila. Infect Immun 72 : 1084-95.

6. Elseviers D, Petrullo LA, Gallagher PJ (1984) Novel E. coli mutants deficient in biosynthesis of 5-methylaminomethyl-2-thiouridine. Nucleic Acids Res 12: 3521-3534.

7. Bregeon D, Colot V, Radman M, Taddei F (2001) Translational misreading: a tRNA modification counteracts a +2 ribosomal frameshift. Genes Dev 15: 2295-306.

8. Cabedo H, Macian F, Villarroya M, Escudero JC, Martinez-Vicente M, Knech $E$, et al (1999) The Escherichia coli trmE (mnmE) gene, involved in tRNA modification, codes for an evolutionarily conserved GTPase with unusual biochemical properties. EMBO J 18: 7063-76.

9. Gong S, Ma Z, Foster JW (2004) The Era-like GTPase TrmE conditionally activates gadE and glutamate-dependent acid resistance in Escherichia coli. Mol Microbiol 54: 948-61.

10. Yim L, Moukadiri I, Bjork GR, Armengod ME (2006) Further insights into the tRNA modification process controlled by proteins MnmE and GidA of Escherichia coli. Nucleic Acids Res 34: 5892-905.

11. Moukadiri I, Prado S, Piera J, Velazquez-Campoy A, Bjork GR, et al. (2009) Evolutionarily conserved proteins MnmE and GidA catalyze the formation of two methyluridine derivatives at tRNA wobble positions. Nucleic Acids Res 37: 7177-93.

12. Shi R, Villarroya M, Ruiz-Partida R, Li Y, Proteau A, Prado S, et al (2009) Structure-function analysis of Escherichia coli MnmG (GidA), a highly conserved tRNA-modifying enzyme. J Bacteriol 191: 7614-9.

13. Osawa T, Ito K, Inanaga H, Nureki O, Tomita K, Numata T (2009) Conserved cysteine residues of GidA are essential for biogenesis of 5-carboxymethylaminomethyluridine at tRNA anticodon. Structure 17: 713-24.

14. Shippy DC, Eakley NM, Lauhon CT, Bochsler PN, Fadl AA (2013) Virulence characteristics of Salmonella following deletion of genes encoding the tRNA modification enzymes GidA and MnmE. Microb Pathog 57: 1-9.

15. White DJ, Merod R, Thomasson B, Hartzell PL (2001) GidA is an FAD-binding protein involved in development of Myxococcus xanthus. Mol Microbiol 42: 50317.

16. Kinscherf TG, Willis DK (2002) Global regulation by gidA in Pseudomonas syringae. J Bacteriol 184: 2281-2286.

17. Gupta R, Gobble TR, Schuster M (2009) GidA posttranscriptionally regulates rhl quorum sensing in Pseudomonas aeruginosa. J Bacteriol 191: 5785-92.

18. Shippy DC, Eakley NM, Bochsler PN, Chopra AK, Fadl AA (2011) Biological and virulence characteristics of Salmonella enterica serovar Typhimurium following deletion of glucose-inhibited division (gidA) gene. Microb Pathog 50 303-13.

19. Shippy DC, Fadl AA (2012) Immunological characterization of a gidA mutant strain of Salmonella for potential use in a live-attenuated vaccine. BMC Microbiol 12: 286.

20. Cho KH, Caparon MG (2008) tRNA modification by GidA/MnmE is necessary for Streptococcus pyogenes virulence: a new strategy to make live attenuated strains. Infect Immun 76: 3176-3186. 